\title{
Vertical elliptic operator for efficient wave propagation in TTI media \\ Umair bin Waheed ${ }^{*}$ and Tariq Alkhalifah \\ King Abdullah University of Science and Technology
}

\section{SUMMARY}

Elliptic wave extrapolation operators require significantly less computational cost than the ones for transversely isotropic (TI) media. However, it does not provide accurate wavefield representation or imaging for the prevalent TI media. We propose a new vertical elliptically anisotropic (VEA) wave equation by decomposing the acoustic TI pseudo-differential wave equation. The decomposition results in a vertical elliptic differential equation and a scalar operator. The new VEA-like wave equation shares the same dispersion relation as that of the original acoustic TI wave equation. Therefore, the kinematic contents are correctly matched to the original equation. Moreover, the proposed decomposition yields better amplitude properties than the isotropic decomposition without increasing the computational load. Therefore, it exhibits better cost versus accuracy tradeoff compared to the isotropic or the tilted elliptic decompositions. We demonstrate with numerical examples that the proposed methodology is numerically stable for complex models and is free from shear-wave artifacts.

\section{INTRODUCTION}

Advanced imaging algorithms, such as reverse time migration (RTM) relies on accurate and efficient forward and backward extrapolation of waves in time. RTM has been a successful tool for imaging strong structural and velocity complexities, such as those in sedimentary regions with steep salt inclusions. However, these methods require solving the usually expensive two way wave equation.

Seismologists have long recognized that in sedimentary rocks wave propagation tend to have an anisotropic behavior. Therefore, taking the anisotropy of the medium into consideration provides more accurate description of wave propagation compared to the isotropic approximation. However, solving the full anisotropic/elastic wave equation is computationally expensive. Therefore, Alkhalifah (2000) proposed an alternative acoustic anisotropic wave equation for transversely isotropic (TI) media by setting the shear-wave velocity along the symmetry direction to zero. This results in a scalar fourth-order pseudo-differential equation that shares almost the same Pwave kinematics as the elastic wave equation, given by the dispersion relation.

Zhou et al. (2006) introduced a $2 \times 2$ system of coupled differential equations based on the acoustic assumption, with better stability features. However, setting the shear wave velocity along the symmetry axis to zero does not completely get rid of the shear waves and we observe shear-wave artifacts in the $\mathrm{qP}$ wave solution. These shear-wave artifacts can cause numerical instability, in addition to causing uncertainty in interpretation. Although these artifacts can be eliminated by applying noise suppressing filters (Zhang et al., 2009; Guan et al., 2011) at each time step; however, it significantly increases the computational load.

Attempting to solve the afore-mentioned issues with the acoustic anisotropic wavefield extrapolation, Alkhalifah et al. (2013) proposed the use of effective isotropic models for wave propagation in anisotropic media. The idea relies on finding a simpler effective model that exhibits the same kinematic wave behavior as the original model. The source-dependent effective isotropic model was generated by embedding the kinematic effects of the anisotropic medium into the isotropic one using the solution to the anisotropic eikonal equation. The effective isotropic model was then used to compute wavefields by solving the significantly cheaper acoustic isotropic wave equation. The resulting wavefield yielded a perfect kinematic match for the fastest arriving wave, however, the later arrivals and the amplitude information suffered from significant inaccuracy (Ibanez-Jacome et al., 2014).

Later, Waheed and Alkhalifah (2014a) improved the kinematic match for later arrivals and amplitude information by using effective elliptic models. However, these methods are expected to yield accurate RTM images only if the dominant imaging energies of the wavefield are present in the first arrival.

Recently, Xu and Zhou (2014) proposed an alternative approach to solve the acoustic anisotropic wave equation. They obtain a new equation by decomposing the pseudo-differential operator into two numerically solvable operators: a Laplacian operator and a scalar operator. This approach requires solving an equation similar to the acoustic isotropic wave equation, thereby significantly reducing the computational load. The approach improved the kinematic match of later arrivals as opposed to the effective isotropic based approximation; nevertheless, the amplitude distortion was evident in the obtained RTM images (Tang et al., 2014).

To improve the amplitude accuracy, Xu et al. (2015) modified the original decomposition, such that the pseudo-differential operator is now decomposed into an elliptic differential operator and a scalar operator. For transversely isotropic media with vertical symmetry axis (VTI), this would mean solving a vertical elliptically anisotropic (VEA) differential operator. However, for tilted transversely isotropic media (TTI), the decomposition would yield a tilted elliptically anisotropic (TEA) differential operator. It is worth noting that there is a significant cost difference between solving the TEA and the VEA wave equations. This cost difference is associated with the mixed partial derivatives arising in the wave equation when tilt is non-zero.

In this abstract, we propose an alternate strategy by decomposition of the pseudo-differential operator into a VEA differential operator and a scalar operator, even when the TI medium exhibits non-zero tilt. In essence, we solve a VEA-like wave equation at each time step; therefore, the cost is equivalent to 


\section{Anisotropic wave propagation}

the isotropic decomposition. We observe that the amplitude match is slightly inferior to the TEA case; however, the cost is significantly lower. Therefore, the proposed decomposition yields a much improved cost versus efficiency trade-off. Furthermore, the wavefield solution does not contain shear-wave artifacts and therefore does not require costly noise-filtering operations. We demonstrate these assertions through tests on synthetic TTI models.

\section{THEORY}

Let $P(x, z, t)$ be the seismic wavefield at location $(x, z)$ at time $t$. Then, the scalar pseudo-differential equation for 2D VTI medium, under the acoustic assumption, is given as (Alkhalifah, 2000):

$$
\begin{aligned}
& \left(\frac{\partial^{2}}{\partial t^{2}}-\frac{v_{0}^{2}}{2}\left(\frac{\partial^{2}}{\partial x^{2}}(1+2 \varepsilon)+\frac{\partial^{2}}{\partial z^{2}}\right.\right. \\
+ & \left.\left.\sqrt{\left(\frac{\partial^{2}}{\partial x^{2}}(1+2 \varepsilon)+\frac{\partial^{2}}{\partial z^{2}}\right)^{2}-8(\varepsilon-\delta) \frac{\partial^{2}}{\partial x^{2}} \frac{\partial^{2}}{\partial z^{2}}}\right)\right) P=0,
\end{aligned}
$$

where $v_{0}$ is the on-axis $\mathrm{qP}$-wave velocity, $\varepsilon$ and $\delta$ are the Thomsen anisotropic parameters (Thomsen, 1986).

In the frequency-wavenumber domain, equation 1 can be written as:

$$
\begin{aligned}
& \left(\omega^{2}-\frac{v_{0}^{2}}{2}\left(k_{x}^{2}(1+2 \varepsilon)+k_{z}^{2}\right.\right. \\
+ & \left.\left.\sqrt{\left(k_{x}^{2}(1+2 \varepsilon)+k_{z}^{2}\right)^{2}-8(\varepsilon-\delta) k_{x}^{2} k_{z}^{2}}\right)\right) \widetilde{P}=0 .
\end{aligned}
$$

Here, $\omega$ denotes the angular frequency, while $k_{x}$ and $k_{z}$ denote the $\mathrm{x}$ - and $\mathrm{z}$-components of spatial wavenumber vector $\mathbf{k}$, respectively, and $\widetilde{P}(\mathbf{k}, \omega)$ is the wavefield in the frequencywavenumber domain.

For TTI media, the spatial wavenumbers need to be computed in a rotated coordinate system. Let $\hat{k}_{x}$, and $\hat{k}_{z}$ be the spatial wavenumbers in the new coordinate frame. They are related to $k_{x}$ and $k_{z}$ through the relation:

$$
\begin{aligned}
& \hat{k}_{x}=k_{x} \cos \theta+k_{z} \sin \theta, \\
& \hat{k}_{z}=-k_{x} \sin \theta+k_{z} \cos \theta,
\end{aligned}
$$

where $\theta$ is the tilt angle that the symmetry axis makes with the vertical. Therefore, equation 2 for TTI media can be written as:

$$
\begin{aligned}
& \left(\omega^{2}-\frac{v_{0}^{2}}{2}\left(\hat{k}_{x}^{2}(1+2 \varepsilon)+\hat{k}_{z}^{2}\right.\right. \\
+ & \left.\left.\sqrt{\left(\hat{k}_{x}^{2}(1+2 \varepsilon)+\hat{k}_{z}^{2}\right)^{2}-8(\varepsilon-\delta) \hat{k}_{x}^{2} \hat{k}_{z}^{2}}\right)\right) \widetilde{P}=0 .
\end{aligned}
$$

For simplification, let us replace the term with the square root by $\beta$ and substitute $\hat{k}_{x}$ and $\hat{k}_{z}$ in the resulting equation to get:

$$
\begin{aligned}
& \left(\omega^{2}-\frac{v_{0}^{2}}{2}\left(\left(k_{x} \cos \theta+k_{z} \sin \theta\right)^{2}(1+2 \varepsilon)\right.\right. \\
+ & \left.\left.\left(-k_{x} \sin \theta+k_{z} \cos \theta\right)^{2}+\beta\right)\right) \widetilde{P}=0,
\end{aligned}
$$

where

$$
\beta=\sqrt{\left(\hat{k}_{x}^{2}(1+2 \varepsilon)+\hat{k}_{z}^{2}\right)^{2}-8(\varepsilon-\delta) \hat{k}_{x}^{2} \hat{k}_{z}^{2}} .
$$

After some algebraic manipulations, equation 5 can be rewritten as:

$$
\begin{aligned}
& \left(\omega^{2}-\frac{v_{0}^{2}}{2}\left(\left((1+2 \varepsilon) \cos ^{2} \theta+\sin ^{2} \theta\right) k_{x}^{2}\right.\right. \\
+ & \left.\left.\left(\cos ^{2} \theta+(1+2 \varepsilon) \sin ^{2} \theta\right) k_{z}^{2}+2 \varepsilon k_{x} k_{z} \sin 2 \theta+\beta\right)\right) \widetilde{P}=0 .
\end{aligned}
$$

Let

$$
\begin{aligned}
& \alpha_{x}=(1+2 \varepsilon) \cos ^{2} \theta+\sin ^{2} \theta, \\
& \alpha_{z}=\cos ^{2} \theta+(1+2 \varepsilon) \sin ^{2} \theta .
\end{aligned}
$$

By substituting $\alpha_{x}$ and $\alpha_{z}$ in equation 7 and doing some algebra, we obtain:

$$
\left(\omega^{2}-\frac{v_{0}^{2}}{2}\left(\alpha_{x} k_{x}^{2}+\alpha_{z} k_{z}^{2}\right)\left(1+\frac{2 \varepsilon k_{x} k_{z} \sin 2 \theta+\beta}{\alpha_{x} k_{x}^{2}+\alpha_{z} k_{z}^{2}}\right)\right) \widetilde{P}=0 .
$$

Let us define the scalar operator to be:

$$
S_{\mathrm{vea}}=\left(1+\frac{2 \varepsilon k_{x} k_{z} \sin 2 \theta+\beta}{\alpha_{x} k_{x}^{2}+\alpha_{z} k_{z}^{2}}\right) .
$$

Then, our VEA-like wave equation in space-time domain can be written in the form of the product of a vertical elliptic differential operator and a scalar operator:

$$
\frac{\partial^{2} P}{\partial t^{2}}=\left(v_{0}^{2} \alpha_{x} \frac{\partial^{2} P}{\partial x^{2}}+v_{0}^{2} \alpha_{z} \frac{\partial^{2} P}{\partial z^{2}}\right) S_{\text {vea }}
$$

This is a non-linear wave equation as the scalar operator $S_{\text {vea }}$ is a function of the wavefield solution. We can obtain $k_{x}$ and $k_{z}$ using the $\mathrm{x}$ - and z-components of $\nabla P$, respectively. Since the anisotropic parameters, and tilt values are readily available, the scalar operator can be conveniently computed at every time step.

Similar to what Xu and Zhou (2014) and Xu et al. (2015) did, the scalar operator here plays the role of matching the dispersion relation of the VEA-like wave equation to the original acoustic wave equation for TI media. Therefore, it ensures correct phase matching. It is equivalent to the idea of effective models that modifies the velocity to obtain correct kinematic match.

However, the amplitude will suffer depending on the magnitude of the correction applied by the scalar operator. Since the correction would be the largest for the isotropic case, the amplitude would suffer the most as observed in the past studies (Waheed and Alkhalifah, 2014b; Wang et al., 2014). The TEA differential operator requires the smallest correction term, and hence its amplitude will be the closest to the original pseudo-differential equation. However, on the downside its cost is significantly higher than the isotropic case due to the presence of the mixed wavenumber term $\left(k_{x} k_{z}\right)$ inside the differential operator. On the contrary, the VEA differential operator markedly improves the amplitude accuracy compared to the isotropic case without increasing the cost. For the case 


\section{Anisotropic wave propagation}

when either of $\varepsilon$ or $\theta$ is zero, the amplitude properties of our approximation will match that of Xu et al. (2015).

We used 2D TTI media for simplicity of illustration. The idea is easily extendable to 3D TTI and tilted orthorhombic media.

\section{ALGORITHM}

The algorithm is simple to implement. At every time step, we execute the following steps:

1. Evaluate the gradient of the wavefield, $\nabla P$.

2. Compute the scalar operator $S_{\text {vea }}$ using equation 10 .

3. Solve equation 11 using a VEA wave equation solver to obtain the wavefield for the next time step.

The computation cost of this scheme is identical to the isotropic decomposition proposed by Xu and Zhou (2014).

\section{NUMERICAL TESTS}

In this section, we test our proposed VEA differential operator in solving the acoustic TTI wave equation. We use pseudo spectral method for computing spatial derivatives and secondorder finite difference for time derivative. The frequency of the Ricker wavelet used is $10 \mathrm{~Hz}$.

First, we consider a $5 \mathrm{~km} \times 5 \mathrm{~km}$ homogeneous TTI model with the following parameter values: $v_{0}=1.8 \mathrm{~km} / \mathrm{s}, \varepsilon=0.2, \delta=$ 0.1 , and $\theta=30^{\circ}$. A grid spacing of $10 \mathrm{~m}$ is used along both the dimensions. Figures 1 shows wavefield snapshots at 0.9 $\mathrm{s}$ using the isotropic (Figures 1(a)), the vertical elliptic (Figures 1(b)), and the tilted elliptic (Figures 1(c)) differential operators. It can be seen that the isotropic operator introduces imbalanced amplitudes, particularly in the direction orthogonal to the symmetry axis. In essence, the isotropic scalar operator modifies the isotropic velocity to match the TTI dispersion relation. This causes amplitude distortion since the anisotropy effect is maximum orthogonal to the symmetry direction. There is a considerably smaller amplitude imbalance for the vertical elliptic operator. The TEA differential operator results in much balanced amplitudes, albeit at a higher computational cost. All the three approaches generate wavefields for only the qP wave and are free from shear-wave artifacts.

Figure 2 shows wavefield profiles for the three operators to compare the amplitude match. We can see that there is a significant amplitude difference between the isotropic and the tilted elliptic operators. However, despite sharing the same computational cost with the isotropic decomposition, the vertical elliptic operator yields better amplitude properties.

Next, we test our algorithm on a portion of the BP TTI model (Shah, 2007) shown in Figure 3. This portion is particularly interesting due to large tilt values as it poses a significant challenge to the vertical elliptic operator. It also contains sharp variations in the tilt angle that have been shown to cause instability in the conventional algorithms. Figure 4 shows wavefield

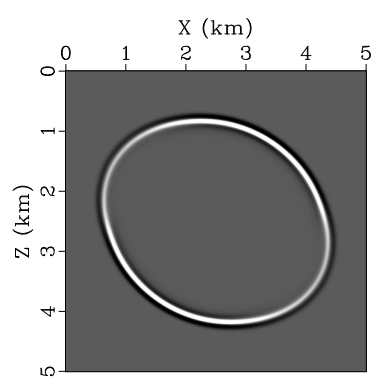

(a)

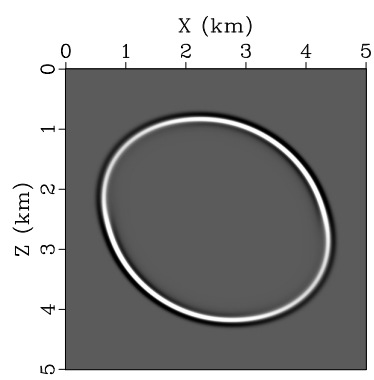

(b)

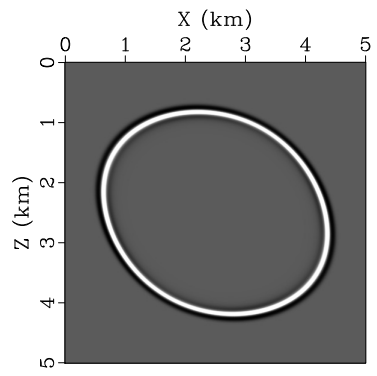

Figure 1: Wavefield snapshots at $0.9 \mathrm{~s}$ for a homogeneous TTI model comparing the wavefield solutions obtained using the isotropic differential operator (a), the vertical elliptic operator (b), and the tilted elliptic operator (c). The source is located at $(2.5 \mathrm{~km}, 2.5 \mathrm{~km})$.

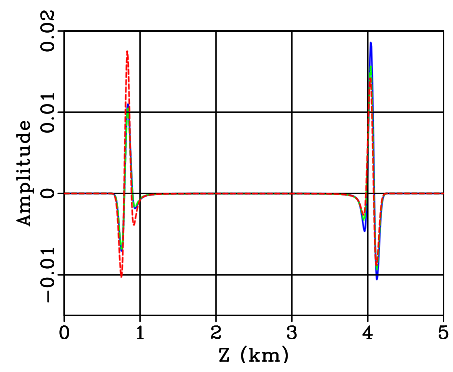

(a)

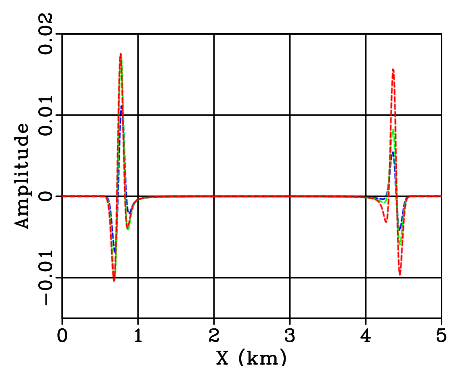

(b)

Figure 2: Wavefield profiles comparing the amplitude properties of the isotropic (blue), the vertical elliptic (green), and the tilted elliptic (red) operators. The plotted profiles are taken at: (a) $\mathrm{X}=2 \mathrm{~km}, \mathrm{t}=0.9 \mathrm{~s}$; (a) $\mathrm{Z}=2.8 \mathrm{~km}, \mathrm{t}=0.9 \mathrm{~s}$. 


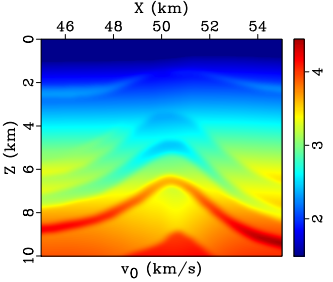

(a)

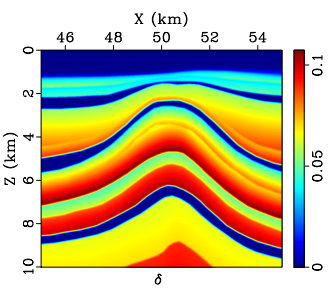

(c)

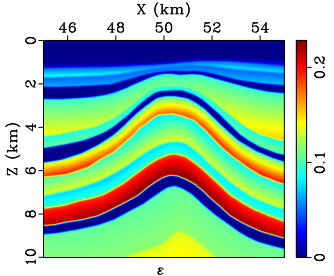

(b)

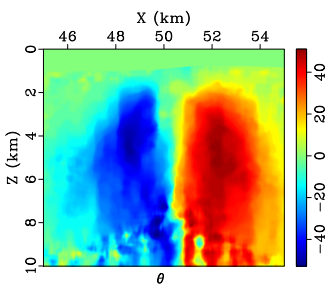

(d)
Figure 3: A portion of the BP TTI model depicting the symmetry axis velocity (a), the Thomsen parameters $\varepsilon(\mathrm{b}), \delta$ (c), and the tilt (d).

snapshots at $1.3 \mathrm{~s}$ using the isotropic (Figure 4(a)), the vertical elliptic (Figure 4(b)), and the tilted elliptic (Figure 4(c)) differential operators, for a source located at $(50 \mathrm{~km}, 5 \mathrm{~km})$. Figure 5 plots wavefield profiles to compare the amplitude properties. As we can see, the phase information is correctly reconstructed by the three operators. However, there are amplitude imbalances, but the VEA operator performs reasonably better than the isotropic one.

\section{CONCLUSIONS}

We propose a decomposition of the acoustic TI pseudodifferential equation into two parts: a vertical elliptic differential equation and a scalar operator. The new VEA-like equation has a similar form as that of the isotropic (Xu and Zhou, 2014) or the tilted elliptic (Xu et al., 2015) decomposition. However, it improves the amplitude properties of the isotropic decomposition without increasing the cost. For TTI models with low tilt values, the amplitude properties will be similar to those of the tilted elliptic differential operator. We note here that the true meaning of amplitude is valid for the elastic case, however, due to our decomposition of the acoustic TI equation, our aim should be to cause minimal amplitude imbalance. Since we are solving a second-order differential equation, the obtained wavefield is free from shear-wave artifacts. Furthermore, the proposed method is stable even for $\varepsilon<\delta$, as opposed to conventional algorithms.

\section{ACKNOWLEDGMENTS}

We are grateful to Dr. Sheng Xu for useful discussions. We acknowledge KAUST for its support.

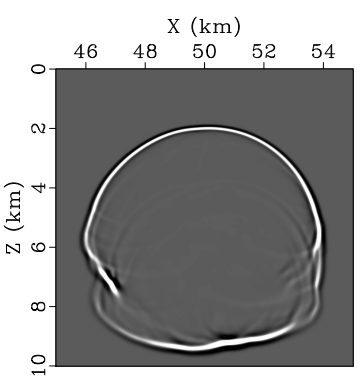

(a)

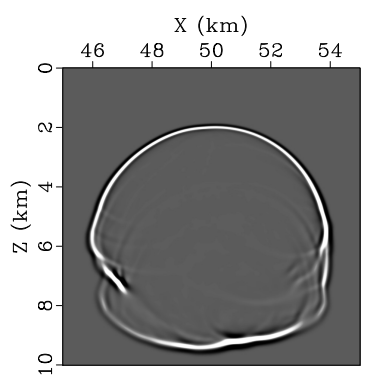

(b)

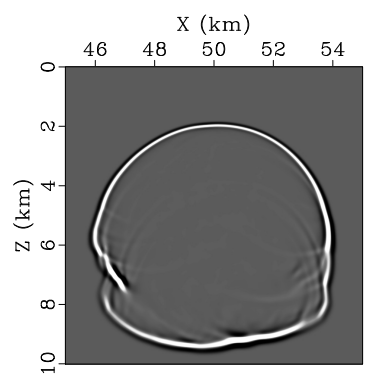

(c)

Figure 4: Wavefield snapshots at $1.3 \mathrm{~s}$ for a portion of the BP TTI model comparing the wavefield solutions obtained using the isotropic differential operator (a), the vertical elliptic operator (b), and the tilted elliptic operator (c). The source is located at $(50 \mathrm{~km}, 5 \mathrm{~km})$.
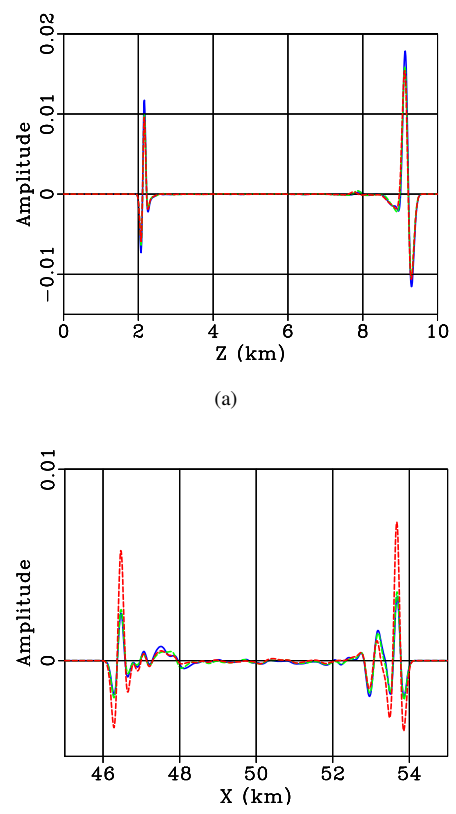

(b)

Figure 5: Wavefield profiles for the BP TTI model comparing the amplitude properties of the isotropic (blue), the vertical elliptic (green), and the tilted elliptic (red) operators. The plotted profiles are taken at: (a) $\mathrm{X}=51.5 \mathrm{~km}, \mathrm{t}=1.3 \mathrm{~s}$; (a) $\mathrm{Z}=53 \mathrm{~km}$, $\mathrm{t}=1.3 \mathrm{~s}$. 
EDITED REFERENCES

Note: This reference list is a copyedited version of the reference list submitted by the author. Reference lists for the 2015 SEG Technical Program Expanded Abstracts have been copyedited so that references provided with the online metadata for each paper will achieve a high degree of linking to cited sources that appear on the Web.

\section{REFERENCES}

Alkhalifah, T., 2000, An acoustic wave equation for anisotropic media: Geophysics, 65, 1239_ 1250. http://dx.doi.org/10.1190/1.1444815.

Alkhalifah, T., X. Ma, U. bin Waheed, and M. Zuberi, 2013, Efficient anisotropic wavefield extrapolation using effective isotropic models: 75th Annual International Conference and Exhibition, EAGE, Extended Abstracts, doi: http://dx.doi.org/10.3997/2214-4609.20130359.

Guan, H., E. Dussaud, B. Denel, and P. Williamson, 2011, Techniques for an efficient implementation of RTM in TTI media: 81st Annual International Meeting, SEG, Expanded Abstracts, doi: http://dx.doi.org/10.1190/1.3627902.

Ibanez-Jacome, W., T. Alkhalifah, and U. bin Waheed, 2014, Effective orthorhombic anisotropic models for wavefield extrapolation: Geophysical Journal International, 198, no. 3, 16531661. http://dx.doi.org/10.1093/gji/ggu229.

Shah, H., 2007, The 2007 BP anisotropic velocity-analysis benchmark: Presented at the 70th Annual International Conference and Exhibition, EAGE.

Tang, B., S. Xu, and H. Zhou, 2014, RTM with pure quasi-P wave equation: 76th Annual International Conference and Exhibition, Extended Abstracts, doi: http://dx.doi.org/10.3997/2214-4609.20141165.

Thomsen, L., 1986, Weak elastic anisotropy: Geophysics, 51, 19541966. http://dx.doi.org/10.1190/1.1442051.

Waheed, U., and T. Alkhalifah, 2014a, Effective elliptic models for efficient wavefield extrapolation in anisotropic media: 76th Annual International Conference and Exhibition, EAGE, Extended Abstracts, doi: http://dx.doi.org/10.3997/2214-4609.20140813.

Waheed, U., and T. Alkhalifah, 2014b, An efficient wave extrapolation method for tilted orthorhombic media using effective ellipsoidal models: Presented at the 84th Annual International Meeting, SEG.

Wang, H., U. bin Waheed, and T. Alkhalifah, 2014, Effective anisotropy through traveltime and amplitude matching: Presented at the 84th Annual International Meeting, SEG, Expanded Abstracts, doi: http://dx.doi.org/10.1190/segam2014-1183.1.

Xu, S., B. Tang, J. Mu, and H. Zhou, 2015, Elliptic decomposition of quasi P-wave equation: 77th Annual International Conference and Exhibition, EAGE, Extended Abstracts, doi:http://dx.doi.org/10.3997/2214-4609.201413134.

$\mathrm{Xu}, \mathrm{S}$., and H. Zhou, 2014, Efficient and accurate algorithm for quasi-P wave propagation: 76th Annual International Conference and Exhibition, EAGE, Extended Abstracts, doi: http://dx.doi.org/10.3997/2214-4609.20141166.

Zhang, J. H., G. Zhang, Y. Zhang, 2009, Removing S-wave noise in TTI reverse time migration: 79th Annual International Meeting, SEG, Expanded Abstracts, doi: http://dx.doi.org/10.1190/1.3255441.

Zhou, H., G. Zhang, and R. Bloor, 2006, An anisotropic acoustic wave equation for VTI media: 68th Annual International Conference and Exhibition, EAGE, Extended Abstracts, doi: http://dx.doi.org/10.3997/2214-4609.201402310. 